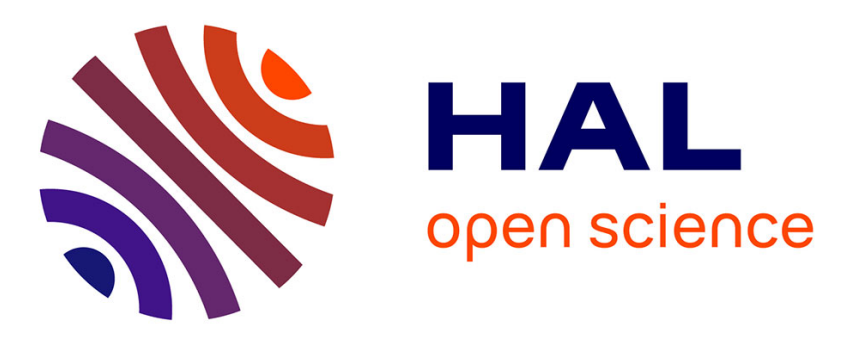

\title{
Delay dependent stability analysis of interval time-delay systems
}

Wenjuan Jiang, Alexandre Kruszewski, Emilia Fridman, Jean-Pierre Richard

\section{To cite this version:}

Wenjuan Jiang, Alexandre Kruszewski, Emilia Fridman, Jean-Pierre Richard. Delay dependent stability analysis of interval time-delay systems. IFAC TDS 2010 - 9th IFAC Workshop on Time Delay Systems, Jun 2010, Prague, Czech Republic. hal-00519491

\section{HAL Id: hal-00519491 \\ https://hal.science/hal-00519491}

Submitted on 20 Sep 2010

HAL is a multi-disciplinary open access archive for the deposit and dissemination of scientific research documents, whether they are published or not. The documents may come from teaching and research institutions in France or abroad, or from public or private research centers.
L'archive ouverte pluridisciplinaire HAL, est destinée au dépôt et à la diffusion de documents scientifiques de niveau recherche, publiés ou non, émanant des établissements d'enseignement et de recherche français ou étrangers, des laboratoires publics ou privés. 


\title{
Delay dependent stability analysis of interval time-delay systems
}

\author{
Wenjuan Jiang* Alexandre Kruszewski ${ }^{* *}$ Emilia Fridman ${ }^{* * *}$ \\ Jean-Pierre Richard ${ }^{* * * *}$ \\ * GIPSA-Lab, CNRS UMR 5216, Rue de la Houille Blanche, BP 46, \\ 38402 Saint Martin d'Heres, France. \\ Nantong University, 9, Seyuan road, Nantong Jiangsu, 226001, China \\ (e-mail: wenjuan.jiang@gipsa-lab.grenoble-inp.fr). \\ ** LAGIS CNRS UMR 8146, Ecole Centrale de Lille, BP 48, 59651 \\ Villeneuve d'Ascq Cedex, France. (e-mail: \\ Alexandre.Kruszewski@ec-lille.fr) \\ *** School of Electrical Engineering, Tel Aviv University, Tel Aviv \\ 69978, Israel.(e-mail: emilia@eng.tau.ac.il) \\ **** LAGIS CNRS UMR 8146, Ecole Centrale de Lille, BP 48, 59651 \\ Villeneuve d'Ascq Cedex, France. \\ Equipe-Projet ALIEN, INRIA(e-mail: jean-pierre.richard@ec-lille.fr)
}

\begin{abstract}
This paper considers interval time-varying delay systems. The time-delay interval is divided into several zones and the systems switch among the different zones. Based on LyapunovKrasovskii functional methods and linear matrix inequality (LMI) techniques, Exponential stability is exploited for every time-delay zone. The global stability of the switched system is guaranteed if some minimum average dwell time conditions are satisfied. Some numerical examples and comparisons with other works show that the methods greatly enlarge the value of maximum upper-bound of time-delay for the systems.
\end{abstract}

Keywords: time-varying delay, switching systems, exponential stability, Lyapunov-Krasovskii functional, LMI.

\section{INTRODUCTION}

A variety of stability and control techniques have been developed for general time delay systems (Niculescu S.I., 2001; Richard J.-P., 2003; Chiasson J. and Loiseau J.J., 2007). Of course, when a delay is introduced into a system, the achievable performance (speed, robustness) highly depends on the delay. In the case of a time-varying delay, the guaranteed performance generally depends on the interval of variation of the delay. To give an example, controlling a system through a communication network induces variable time delays and the resulting performance depends on the quality of service (QoS) the network can offer (Jiang W.-J. et al., 2008; Seuret A. et al., 2006).

In order to reduce the conservatism, some authors used the piecewise analysis method which consist in study the system for smaller delay intervals with a common Lyapunov-Krasovskii Functional (LKF) (Yue D. et al., 2008). Another approach is consider the system as a switching system in which each subsystem has a different delay. These delays belong to smaller intervals.

Switched systems are dynamical hybrid systems consisting of a family of continuous-time subsystems and a logical rule that orchestrates the switching between them (Liberzon D., 2003; Hirche S. et al., 2006). In general, the stability of the subsystems themselves are not sufficient for the stability of the overall system. A lot of studies have dealt with the stability analysis and design of switched systems (Liberzon D. et al., 1999; Hespanha J.P. and Morse A.S., 1999; Xie G. and Wang L., 2005; Chen C.-C. et al., 2006).

Single (Sun Y.G. and Wang L., 2006; Jiang W.-J. et al., 2008) and multiple LKF analysis methods are the most frequently applied in the stabilization of the whole system. A common LKF can ensure the stability with arbitrary switching but it does not always exist. As an alternative, dwell-time based switching is considered in (Hespanha J.P. and Morse A.S., 1999) but time delay is not considered, while in (Yan P. and Özbay H., 2008; Chen C.-C. et al., 2006), the time delay is a constant one.

In this paper, a linear switched time-delay system is considered. Multiple LKF are used in the stability analysis. The time-delay interval is divided into several ones to reduce conservativeness. In this means, the maximum timedelay can be enlarged. The minimum average dwell time is considered to guarantee the global stability of the switched system. The LMI (Linear Matrix Inequalities) conditions are derived following the idea of (Park P.G. and Ko. J.W., 2007) (using the convex approach). The first part introduces some sufficient conditions for exponential stability of linear systems with interval time-varying delay. Our main results are presented in the second part, where the linear time-delay system is analyzed as a switched system and where sufficient condition for the global stability are given 
by using average dwelling time. The last part gives some examples and comparisons with other works.

Notation: Throughout the paper the superscript ' $T$ ' stands for matrix transposition, $\mathcal{R}^{n}$ denotes the $n$ dimensional Euclidean space with vector norm $\|\cdot\|, \mathcal{R}^{n \times m}$ is the set of all $n \times m$ real matrices, and the notation $P>0$, for $P \in \mathcal{R}^{n \times n}$ means that $P$ is symmetric and positive definite. The symmetric elements of the symmetric matrix will be denoted by $* . L_{2}$ is the space of square integrable functions $v:[0, \infty) \rightarrow R^{n}$ with the norm $\|v\|_{L_{2}}=\left[\int_{0}^{\infty}\|v(t)\|^{2} d t\right]^{1 / 2}$.

\section{EXPONENTIAL STABILITY OF INTERVAL TIME-DELAY SYSTEMS}

For the sake of simplicity, the LMI conditions are first derived for exponential stability of a linear time-delay system without switches.

Consider a linear time-delay system:

$$
\dot{x}(t)=A x(t)+A_{1} x(t-\tau(t))
$$

with $A$ stable and $\tau(t)$, a fast varying interval delay with the bounds as $\left[h_{1}, h_{2}\right]$.

In the following, the notation $x_{t}$ stands for $x(t)$. Applying the following LKFs (Fridman E. and Yury Orlov, 2009):

$$
\begin{aligned}
& V\left(t, x_{t}, \dot{x}_{t}\right)=x^{T}(t) P x(t) \\
& +\sum_{i=0}^{1} \int_{t-h_{i+1}}^{t-h_{i}} e^{2 \alpha(s-t)} x^{T}(s) S_{i} x(s) d s \\
& +\sum_{i=0}^{1}\left(h_{i+1}-h_{i}\right) \int_{-h_{i+1}}^{-h_{i}} \int_{t+\theta}^{t} e^{2 \alpha(s-t)} \dot{x}^{T}(s) R_{i} \dot{x}(s) d s d \theta
\end{aligned}
$$

where $h_{0}=0, P>0$ and $R_{i}, S_{i} \geq 0$. Saying that the system (1) is exponentially stable means that there exist a positive function $W(t, x(t), \dot{x}(t))$ such that:

$$
W\left(t, x_{t}, \dot{x}_{t}\right) \leq e^{-2 \alpha\left(t-t_{0}\right)} W\left(t, x_{t_{0}}, \dot{x}_{t_{0}}\right)
$$

It can be ensured by using the LKFs (2) and by checking if:

$$
\dot{V}\left(t, x_{t}, \dot{x}_{t}\right)+2 \alpha V\left(t, x_{t}, \dot{x}_{t}\right)<0
$$

After the differentiation of the function (2), the system is exponentially stable if:

$$
\begin{aligned}
& \dot{V}\left(t, x_{t}, \dot{x}_{t}\right)+2 \alpha V\left(t, x_{t}, \dot{x}_{t}\right) \leq 2 x^{T}(t) P \dot{x}(t) \\
& +2 \alpha x^{T}(t) P x(t)+\dot{x}^{T}(t)\left[\sum_{i=0}^{1}\left(h_{i+1}-h_{i}\right)^{2} R_{i}\right] \dot{x}(t) \\
& -\sum_{i=0}^{1}\left(h_{i+1}-h_{i}\right) e^{-2 \alpha h_{i+1}} \int_{t-h_{i+1}}^{t} \dot{x}^{T}(s) R_{i} \dot{x}(s) d s \\
& +\sum_{i=0}^{1} x\left(t-h_{i}\right)^{T} e^{-2 \alpha h_{i}} S_{i} x\left(t-h_{i}\right) \\
& -\sum_{i=0}^{1} e^{-2 \alpha h_{i+1}} x^{T}\left(t-h_{i+1}\right) S_{i} x\left(t-h_{i+1}\right) .
\end{aligned}
$$

Noticing that:

$$
\begin{aligned}
\int_{t-h_{1}}^{t-h_{0}} \dot{x}^{T}(s) R_{i} \dot{x}(s) d s & =\int_{t-h_{1}}^{t-h_{0}} \dot{x}^{T}(s) R_{0} \dot{x}(s) d s \\
& +\int_{t-\tau(t)}^{t-\tau(t)} \dot{x}^{T}(s) R_{0} \dot{x}(s) d s
\end{aligned}
$$

and applying the Jensen's inequality (Gu K. et al., 2003)

$$
\begin{aligned}
& \int_{t-h_{1}}^{t-h_{0}} \dot{x}^{T}(s)\left[\left(h_{2}-h_{1}\right) R_{0}\right] \dot{x}(s) d s \\
& \geq \int_{t-h_{1}}^{t-h_{0}} \dot{x}^{T}(s) d s R_{0} \int_{t-h_{1}}^{t-h_{0}} \dot{x}(s) d s \\
& t-h_{1} \\
& \int_{t-\tau(t)}^{t-h_{1}} \dot{x}^{T}(s)\left(h_{2}-h_{1}\right) R_{j} \dot{x}(s) d s \\
& \geq \frac{h_{2}-h_{1}}{\tau-h_{1}} \int_{t-\tau(t)}^{t-h_{1}} \dot{x}^{T}(s) d s R_{1} \int_{t-\tau(t)}^{t-h_{1}} \dot{x}(s) d s, \\
& t-\tau(t) \\
& \int_{t-h_{2}} \dot{x}^{T}(s)\left[\left(h_{2}-h_{1}\right) R_{1}\right] \dot{x}(s) d s \\
& \geq \frac{h_{2}-h_{1}}{h_{2}-\tau} \int_{t-h_{2}}^{t-\tau(t)} \dot{x}^{T}(s) d s R_{1} \int_{t-h_{2}}^{t-\tau(t)} \dot{x}(s) d s .
\end{aligned}
$$

Here for $\tau \rightarrow h_{1}$ we understand by

$$
\begin{aligned}
\frac{1}{\tau(t)-h_{1}} \int_{t-\tau(t)}^{t-h_{1}} \dot{x}(s) d s & =\lim _{\tau \rightarrow h_{1}} \frac{1}{\tau(t)-h_{1}} \int_{t-\tau(t)}^{t-h_{1}} \dot{x}(s) d s \\
& =\dot{x}\left(t-h_{1}\right)
\end{aligned}
$$

For $h_{2}-\tau(t) \rightarrow 0$ the vector $\frac{1}{h_{2}-\tau(t)} \int_{t-h_{2}}^{t-\tau(t)} \dot{x}(s) d s$ is defined similarly as $\dot{x}\left(t-h_{2}\right)$.

Denoting

$$
\begin{aligned}
& v_{1}=\frac{1}{\tau-h_{1}} \int_{t-\tau(t)}^{t-h_{1}} \dot{x}(s) d s, \\
& v_{2}=\frac{1}{h_{2}-\tau} \int_{t-h_{2}}^{t-\tau(t)} \dot{x}(s) d s,
\end{aligned}
$$

we obtain 


$$
\begin{aligned}
& \dot{V}\left(t, x_{t}, \dot{x}_{t}\right)+2 \alpha V\left(t, x_{t}, \dot{x}_{t}\right) \leq 2 x^{T}(t) P \dot{x}(t) \\
& \left.+2 \alpha x^{T}(t) P x(t)+\dot{x}^{T}(t) \sum_{i=0}^{1}\left(h_{i+1}-h_{i}\right)^{2} R_{i}\right) \dot{x}(t) \\
& +\sum_{i=0}^{1} e^{-2 \alpha h_{i}} x^{T}\left(t-h_{i}\right) S_{i} x\left(t-h_{i}\right) \\
& -\sum_{i=0}^{1} e^{-2 \alpha h_{i+1}} x^{T}\left(t-h_{i+1}\right) S_{i} x\left(t-h_{i+1}\right) \\
& -\left[x(t)-x\left(t-h_{1}\right)\right]^{T} e^{-2 \alpha h_{1}} R_{0}\left[x(t)-x\left(t-h_{1}\right)\right] \\
& -\left(\tau-h_{1}\right)\left(h_{2}-h_{1}\right) v_{1}^{T} e^{-2 \alpha h_{2}} R_{1} v_{1} \\
& -\left(h_{2}-\tau\right)\left(h_{2}-h_{1}\right) v_{2}^{T} e^{-2 \alpha h_{2}} R_{1} v_{2} .
\end{aligned}
$$

We note that in the latter bound we can substitute for $\tau$ its upper bound $h_{2}$.

We insert free-weighting $n \times n$-matrices (He Y. et al., 2004, $2007)$ by adding the following expressions to $\dot{V}$ :

$$
\begin{aligned}
0 & =2\left[x^{T}(t) Y_{1}^{T}+\dot{x}^{T}(t) Y_{2}^{T}+x^{T}(t-\tau) T_{1}^{T}\right]\left[-x\left(t-h_{1}\right)\right. \\
& \left.+x(t-\tau)+\left(\tau-h_{1}\right) v_{1}\right], \\
0 & =2\left[x^{T}(t) Z_{1}^{T}+\dot{x}^{T}(t) Z_{2}^{T}\right]\left[x\left(t-h_{2}\right)\right. \\
& \left.+\left(h_{2}-\tau\right) v_{2}-x(t-\tau)\right] .
\end{aligned}
$$

We use further the descriptor method (Fridman E., 2001), where the right-hand side of the expression

$$
0=2\left[x^{T}(t) P_{2}^{T}+\dot{x}^{T}(t) P_{3}^{T}\right]\left[A x(t)+A_{1} x(t-\tau(t))-\dot{x}(t)\right],
$$

with some $n \times n$-matrices $P_{2}, P_{3}$ is added into the righthand side of (8).

Setting $\eta(t)=\operatorname{col}\left\{x(t), \dot{x}(t), x\left(t-h_{1}\right), x\left(t-h_{2}\right), v_{1}, v_{2}, x(t-\right.$ $\tau)\}$, we obtain that along the time-delay system (1)

$$
\dot{V}\left(t, x_{t}, \dot{x}_{t}\right)+2 \alpha V\left(t, x_{t}, \dot{x}_{t}\right) \leq \eta^{T}(t) \Phi \eta(t)<0,
$$

if the LMI (12) is feasible,

where $\tau=h_{2}$ and

$$
\begin{aligned}
& \tilde{R}_{i}=e^{-2 \alpha h_{i+1}} R_{i}, \tilde{S}_{i}=e^{-2 \alpha h_{i+1}} S_{i}, \hat{S}_{i}=e^{-2 \alpha h_{i}} S_{i}, \\
& \Phi_{11}=A^{T} P_{2}+P_{2}^{T} A+S_{0}-e^{-2 \alpha h_{1}} R_{0}+2 \alpha P, \\
& \Phi_{12}=P-P_{2}^{T}+A^{T} P_{3}, \\
& \Phi_{22}=-P_{3}-P_{3}^{T}+\sum_{i=0}^{1}\left(h_{i+1}-h_{i}\right)^{2} R_{i}, \\
& \Phi_{16}=P_{2}^{T} A_{1}, \Phi_{26}=P_{3}^{T} A_{1}, \phi_{3}=-\left(\tilde{S}_{0}+\tilde{R}_{0}-\hat{S}_{1}\right), \\
& \phi_{4}=-\tilde{S}_{1}, \phi_{5}=-\left(h_{2}-h_{1}\right)\left(\tau-h_{1}\right) \tilde{R}_{1}, \\
& \phi_{6}=-\left(h_{2}-h_{1}\right)\left(h_{2}-\tau\right) \tilde{R}_{1} .
\end{aligned}
$$

The latter condition leads for $\tau \rightarrow h_{1}$ and for $\tau \rightarrow h_{2}$ to the following LMIs (13) and (14), where we deleted the zero column and the zero row: Denote by $\eta_{i}(t)=$ $\operatorname{col}\left\{x(t), \dot{x}(t), x\left(t-h_{1}\right), x\left(t-h_{2}\right), v_{i}, x(t-\tau)\right\}, i=1,2$.

Then (13), (14) imply (11) because

$$
\begin{aligned}
& \frac{h_{2}-\tau}{h_{2}-h_{1}} \eta_{2}^{T}(t) \Psi_{1} \eta_{2}(t)+\frac{\tau-h_{1}}{h_{2}-h_{1}} \eta_{1}^{T}(t) \Psi_{2} \eta_{1}(t) \\
& =\eta^{T}(t) \Psi \eta(t)<0 .
\end{aligned}
$$

Thus, $\Psi$ is convex in $\tau \in\left[h_{1}, h_{2}\right]$ (Park P.G. and Ko. J.W., 2007).

if the four LMIs (13) and (14) are feasible, thus, the following result is obtained.
Lemma 1. Let there exist $n \times n$-matrices $P>0, R_{i}>$ $0, S_{i}>0, i=0,1, P_{2}, P_{3}, Y_{1}, Y_{2}, T_{1}, T_{2}, Z_{1}$ and $Z_{2}$ such that the four LMIs (13) and (14) with notations given in (15) are feasible. Then the switched system (1) is exponentially stable for delay $\tau \in\left[h_{1}, h_{2}\right]$ with a decay rate $\alpha$.

\section{AVERAGE DWELL-TIME FOR SWITCHED TIME-DELAY INTERVALS}

In order to reduce the conservatism, one would like to use multiple LKF on smaller delay intervals. To achieves this, the system (1) is rewritten as a switching system:

$$
\dot{x}(t)=A x(t)+\sum_{i=0}^{N} \beta_{i} A_{1} x\left(t-\tau_{i}(t)\right),
$$

where $\beta_{i} \in\{0,1\}$ and $\sum_{i=0}^{N} \beta_{i}=1, N$ corresponds to the number of the time-delay intervals.

As multiple LKF are used for different delay intervals, arbitrary switching cannot be obtained. So a certain criteria of minimum dwell time will be considered to guarantee the global stability(Liberzon D., 2003).

Here, to simplify the problem, the time-delay interval is equally divided into $N$ smaller intervals. Each one is considered for a mode of the switched system. Then, the bounds of the intervals can be written as $\left[\nu_{i}, \nu_{i+1}\right], i \in$ $\{1, \ldots, N\}$, with $\nu_{0}=0, \nu_{1}=h_{1}, \nu_{N+1}=h_{2}$ and $\bigcup_{i=1}^{N}\left[\nu_{i}, \nu_{i+1}\right]=\left[h_{1}, h_{2}\right]$.

The stability of the system (1) equals to the global stability of the one of (16). Two methods are usually applied for verifying stability of switched systems: one is to find the common LKF and the other is to use different LKFs but with minimum dwell time for each mode. The common LKF cannot always be found, and in case of exponential stability, only the same value of $\alpha$ can be obtained, i.e., the performance in each mode cannot be taken into account and the performance cannot be better in the case of small time-delay. Not using a common LKF reduces the conservatism as each mode can have a completely different behavior. This reduction allows, in most cases, getting better performances or robustness. So, here the latter method is applied.

The stability analysis is performed by using different LKFs for each time-delay interval. In order to make the LKFs comparable, their structure are chosen as follows:

$$
\begin{aligned}
& V_{i}\left(t, x_{t}, \dot{x}_{t}\right)=x^{T}(t) P_{i} x(t) \\
& +\sum_{j=0}^{N} \int_{t-\nu_{j+1}}^{t-\nu_{j}} e^{2 \alpha_{i}(s-t)} x^{T}(s) S_{j i} x(s) d s \\
& +\sum_{j=0}^{N}\left(\nu_{j+1}-\nu_{j}\right) \int_{-\nu_{j+1}}^{-\nu_{j}} \int_{t+\theta}^{t} e^{2 \alpha_{i}(s-t)} \dot{x}^{T}(s) R_{j i} \dot{x}(s) d s d \theta
\end{aligned}
$$

where $i$ represents the mode, $V_{i}$ for $\tau_{i} \in\left[\nu_{i}, \nu_{i}+r\right]$.

These LKFs consider the delay over $N$ zones. Note that if set $\nu_{1}=h_{1}, N=1$, then the previous LKFs (2) are recovered for a time-delay in $\tau(t) \in\left[h_{1}, h_{2}\right]$.

Therefore, to verify the stability of system (1) can be divided into the following two small problems: 


$$
\begin{aligned}
& \Psi=\left[\begin{array}{ccccccc}
\Phi_{11}-(1-\dot{\tau}) X & \Phi_{12} & \tilde{R}_{0}-Y_{1}^{T} & Z_{1}^{T} & \left(\tau-h_{1}\right) Y_{1}^{T} & \left(h_{2}-\tau\right) Z_{1}^{T} & Y_{1}^{T}-Z_{1}^{T}+\Phi_{16} \\
* & \Phi_{22} & -Y_{2}^{T} & Z_{2}^{T} & \left(\tau-h_{1}\right) Y_{2}^{T} & \left(h_{2}-\tau\right) Z_{2}^{T} & Y_{2}^{T}-Z_{2}^{T}+\Phi_{26} \\
* & * & \phi_{3} & 0 & 0 & 0 & -T_{1} \\
* & * & * & \phi_{4} & 0 & 0 & 0 \\
* & * & * & * & \phi_{5} & 0 & \left(\tau-h_{1}\right) T_{1} \\
* & * & * & * & * & \phi_{6} & 0 \\
* & * & * & * & * & * & T_{1}+T_{1}^{T}
\end{array}\right]<0, \\
& \Psi_{1}=\left[\begin{array}{cccccc}
\Phi_{11} & \Phi_{12} & \tilde{R}_{0}-Y_{1}^{T} & Z_{1}^{T} & \left(h_{2}-h_{1}\right) Z_{1}^{T} & Y_{1}^{T}-Z_{1}^{T}+\Phi_{16} \\
* & \Phi_{22} \mid \tau=h_{1} & -Y_{2}^{T} & Z_{2}^{T} & \left(h_{2}-h_{1}\right) Z_{2}^{T} & Y_{2}^{T}-Z_{2}^{T}+\Phi_{26} \\
* & * & \phi_{3} & 0 & 0 & -T_{1} \\
* & * & * & \phi_{4} & 0 & 0 \\
* & * & * & * & \phi_{6 \mid \tau=h} & 0 \\
* & * & * & * & * & T_{1}+T_{1}^{T}
\end{array}\right]<0, \\
& \Psi_{2}=\left[\begin{array}{cccccc}
\Phi_{11} & \Phi_{12} & \tilde{R}_{0}-Y_{1}^{T} & Z_{1}^{T} & \left(h_{2}-h_{1}\right) Y_{1}^{T} & Y_{1}^{T}-Z_{1}^{T}+\Phi_{16} \\
* & \Phi_{22} \mid \tau=h_{2} & -Y_{2}^{T} & Z_{2}^{T} & \left(h_{2}-h_{1}\right) Y_{2}^{T} & Y_{2}^{T}-Z_{2}^{T}+\Phi_{26} \\
* & * & \phi_{3} & 0 & 0 & -T_{1} \\
* & * & * & \phi_{4} & 0 & 0 \\
* & * & * & * & \phi_{5} \mid \tau=h_{2} & \left(h_{2}-h_{1}\right) T_{1} \\
* & * & * & * & * & T_{1}+T_{1}^{T}
\end{array}\right]<0 .
\end{aligned}
$$

(1) Guarantee the stability of the system in each time-delay interval. Apply the Theorem 1 to each time-delay interval, the maximum value of exponential decrease decay, i.e. $\alpha_{i}$, of each mode can be obtained.

(2)According to the theory of (Hespanha J.P. and Morse A.S., 1999) and (Zhai G. et al., 2001), an average dwell time has to be calculated to guarantee the global system stability.

Consider during a period of time as $t_{a}$ as showed in the Fig. 1, $N^{\prime}$ denotes the number of switching over the time of $t_{a}$. For generalizing the problem, we consider $N^{\prime} \geq N$.

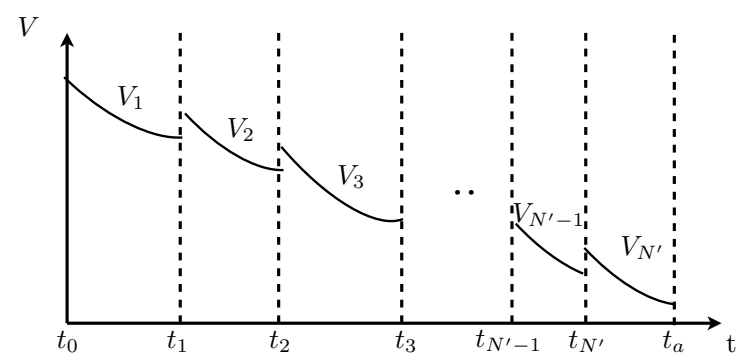

Fig. 1. Switching time-delay intervals

Decreasing sequence in Fig. 1 implies that there exits $\mu_{i}, i \in\left\{1, \ldots, N^{\prime}\right\}$, the smaller positive real such that:

$$
V_{i+1}\left(t, x_{t}, \dot{x}_{t}\right) \leq \mu_{i} V_{i}\left(t, x_{t}, \dot{x}_{t}\right)
$$

This is implied by the following conditions:

$$
\begin{aligned}
& P_{i+1} \leq \mu_{i} P_{i} \\
& e^{-2 \alpha_{i+1} \nu_{j}} S_{j(i+1)} \leq \mu_{i} e^{-2 \alpha_{i} \nu_{j}} S_{j i}, \\
& e^{-2 \alpha_{i+1} \nu_{j}} R_{j(i+1)} \leq \mu_{i} e^{-2 \alpha_{i} \nu_{j}} R_{j i}
\end{aligned}
$$

with $j \in\left\{1,2, \ldots, N^{\prime}\right\}$.

If the LMIs conditions above are satisfied, one have: $V_{i}\left(t, x_{t}, \dot{t}_{t}\right) \leq e^{-2 \alpha_{i}\left(t-t_{0}\right)} V_{i}\left(t_{0}, x_{t_{0}}, \dot{x}_{t_{0}}\right)$ for each interval of time-delay. According to these conditions, the smaller positive $\mu_{i}$ can be calculated.

Notice that:

$$
\begin{aligned}
& V_{N^{\prime}}\left(t_{a}, x_{t_{a}}, \dot{x}_{t_{a}}\right) \leq e^{-2 \alpha_{N^{\prime}}\left(t_{a}-t_{N^{\prime}}\right)} V_{N^{\prime}}\left(t_{N^{\prime}}, x_{t_{N^{\prime}}}, \dot{x}_{t_{N^{\prime}}}\right) \\
& \leq \mu_{N^{\prime}} e^{-2 \alpha_{N^{\prime}}\left(t_{a}-t_{N^{\prime}}\right)} V_{N^{\prime}-1}\left(t_{N^{\prime}}, x_{t_{N^{\prime}}}, \dot{x}_{t_{N^{\prime}}}\right) \\
& \leq \cdots \\
& \leq \mu_{1} \mu_{2} \cdots \mu_{N^{\prime}} e^{-2 \alpha_{1} t_{1}} e^{-2 \alpha_{2}\left(t_{2}-t_{1}\right)} \cdots e^{-2 \alpha_{N^{\prime}}\left(t_{a}-t_{N^{\prime}}\right)} \\
& V_{1}\left(t_{0}, x_{t_{0}}, \dot{x}_{t_{0}}\right)
\end{aligned}
$$

$\mu_{1} \mu_{2} \cdots \mu_{N^{\prime}} e^{-2 \alpha_{1} t_{1}} e^{-2 \alpha_{2}\left(t_{2}-t_{1}\right)} \cdots e^{-2 \alpha_{N^{\prime}}\left(t_{a}-t_{N^{\prime}}\right)} \leq 1$ is sufficient to prove the switching stability for a given period of time $t_{a}$ showed in Fig. 1, so the minimum average dwell time for the system has to satisfy the following condition:

$$
\begin{aligned}
& \left(\alpha_{1}-\alpha_{2}\right) t_{1}+\left(\alpha_{2}-\alpha_{3}\right) t_{2}+\cdots+\alpha_{N^{\prime}} t_{a} \\
& \geq 0.5 \ln \left(\mu_{1} \mu_{2} \cdots \mu_{N^{\prime}}\right) .
\end{aligned}
$$

\section{NUMERICAL EXAMPLES}

In this section, we use two numerical examples as the same as in (He Y. et al., 2004) to show our results. To compare with other methods, we here consider the stability conditions, i.e., in lemma (1), $\alpha=0$.

Example 1. Consider the system (1) with

$$
A=\left[\begin{array}{cc}
-2 & 0 \\
0 & -0.9
\end{array}\right], A_{1}=\left[\begin{array}{cc}
-1 & 0 \\
-1 & -1
\end{array}\right] \text {. }
$$

As listed in the table Fig. 4 , when $h_{1}=0$, our result greatly enlarge the value of maximum upper-bound of time-delay for the system. While considering for the case of interval variable time-delay, the comparison with the methods of (Jiang X. and Han Q. L., 2005) and (He Y. et al., 2007) shows the merits of our methods. Note that when $h_{1}=0$, the result coincides with (Park P.G. and Ko. J.W., 2007).

To show the merits of using several intervals of timedelay, we take the same example with two intervals as suggested in (Yue D. et al., 2008). For simplicity, two intervals here are considered, i.e., the total interval is averaged divided into two smaller ones. Then the result in table 4 can be obtained. It is obvious that using multiple LKF reduces conservatism compared with common LKF (Yue D. et al., 2008), but a certain minimum dwell time has to be considered. So, for calculating the minimum dwell time, we divide the interval into two smaller ones. As showed in the equation 21, there is a compromise between the value of $\alpha$ and $\mu$. For this example, we consider the time-delay interval as $[h 1, h 2]=[0,2.0]$, the best solution 


\begin{tabular}{cccccccc}
\hline Method & $h_{1}$ & 0 & 1 & 2 & 3 & 4 & 4.4697 \\
\hline (Jiang X. and Han Q. L., 2005) & $h_{2}$ & 1.01 & 1.64 & 2.39 & 3.20 & 4.06 & - \\
(He Y. et al., 2007) & $h_{2}$ & 1.34 & 1.74 & 2.43 & 3.22 & 4.07 & 4.47 \\
(Park P.G. and Ko. J.W., 2007) & $h_{2}$ & 1.86 & - & - & - & - & - \\
Lemma 1 & $h_{2}$ & 1.86 & 2.06 & 2.61 & 3.31 & 4.09 & 4.47 \\
\hline
\end{tabular}

Fig. 2. Allowable upper bound of $h_{2}$ with given $h_{1}$

\begin{tabular}{ccccccc}
\hline Method & $\nu_{1}$ & 0 & 0.5 & 1.0 & 1.5 & 2.0 \\
\hline (Yue D. et al., 2008) & $\nu_{3}$ & 1.98 & 2.05 & 2.16 & 2.37 & 2.64 \\
our result & $\nu_{3}$ & 2.08 & 2.34 & 2.58 & 2.81 & 3.05 \\
\hline
\end{tabular}

Fig. 3. Allowable upper bound with switching delays

\begin{tabular}{cccccccc}
\hline Method & $h_{1}$ & 0 & 0.3 & 0.5 & 0.8 & 1 & 2 \\
\hline (Jiang X. and Han Q. L., 2005) & $h_{2}$ & 0.67 & 0.91 & 1.07 & 1.33 & 1.50 & 2.39 \\
(He Y. et al., 2007) & $h_{2}$ & 0.77 & 0.94 & 1.09 & 1.34 & 1.51 & 2.40 \\
Lemma 1 & $h_{2}$ & 1.06 & 1.24 & 1.38 & 1.6 & 1.75 & 2.58 \\
\hline
\end{tabular}

Fig. 4. Allowable upper bound of $h_{2}$ with given $h_{1}$

is $\alpha_{1}=0.46, \alpha_{2}=0.01, \mu_{1}=\mu_{2}=15$. According to the equation 21 , the minimum average dwell time can be calculated for a given period of time.

Example 2. Consider the system (1) with

$$
A=\left[\begin{array}{cc}
0 & 1 \\
-1 & -2
\end{array}\right], A_{1}=\left[\begin{array}{cc}
0 & 0 \\
-1 & 1
\end{array}\right] .
$$

In the table Fig. 4 , for given lower bounds $h_{1}$ of variable time-delay, the corresponding maximum values of upper bounds $h_{2}$ are given by considering different methods to guarantee the stability of the system. The comparison shows that our results are much less conservative than the other methods.

As we can find in the two examples, when the upper bound of time-delay becomes bigger, considering an unique interval, it becomes very difficult to find a LKF to guarantee the stability for the system. Even that Lemma 1 has greatly removed the conservation for the result, but for the greater delay, the result is not satisfied. The method of dividing the one interval into several ones enlarges the bounds of time-delays.

\section{CONCLUSION}

In this paper, the delay-dependent stability condition is given for interval LTI time-delay system based on the piecewise analysis method and LKF method. The timedelay interval is divided into several smaller ones and the system switches according to the bounds of each interval. Using multiple LKF, exponential stability of each mode is obtain and the global stability is guaranteed by the average dwell time. For sake of simplicity, the delay interval is equally divided, but for some special cases when the timedelay varies much around the bounds of the intervals, an oscillation will be brought to the system. So, some techniques for dividing the time-delay interval can be further developed.

\section{REFERENCES}

Chen C.-C., Hirche S., and Buss M. (2006). Towards quality-of-service control of networked control systems: a switched time delay systems approach. Proceedings of the 2006 IEEE International Conference on Control Applications.

Chiasson J. and Loiseau J.J. (2007). Applications of time delay systems, volume 352. Springer.

Fridman E. (2001). New lyapunov-krasovskii functionals for stability of linear retarded and neutral type systems. Systems \& Control Letters, 43, 309-319.

Fridman E. and Yury Orlov (2009). Exponential stability of linear distributed parameter systems with timevarying delays. Automatica.

Gu K., Kharitonov V., and Chen J. (2003). Stability of time-delay systems. Birkhauser: Boston.

He Y., Wang Q-G., Lin C., and Wu M. (2007). Delayrange-dependent stability for systems with time-varying delay. Automatica, 43(2), 371-376.

He Y., Wu M., She J.H., and Liu G.P. (2004). Parameterdependent lyapunov functional for stability of timedelay systems with polytopic-type uncertainties. IEEE Transactions on Automatic Control, 49, 828-832.

Hespanha J.P. and Morse A.S. (1999). stability of switched systems with average dwell-time. Proceedings of the $38^{\text {th }}$ Conference on Decision \& Control, 2655-2660.

Hirche S., Chen C.-C, and Buss M (2006). Performance oriented control over networks -switching controllers and switched time delay-. Proceedings of the 45th IEEE Conference on Decision \& Control.

Jiang W.-J., Kruszewski A., Richard J.-P., and Toguyeni A. (2008). A gain scheduling strategy for the control and estimation of a remote robot via internet. The $27 t h$ Chinese Control Conference.

Jiang X. and Han Q. L. (2005). On h control for linear systems with interval time-varying delay. Automatica, 41, 2099-2106.

Liberzon D. (2003). Switching in Systems and Control. Birkhuser.

Liberzon D., Hespanha J.P., and Morse A.S. (1999). Stability of switched systems: a lie-algebraic condition. Systems Control Lett., 37, 117-122.

Niculescu S.-I. (2001). Delay effects on stability: a robust control approach, volume 269. Springer.

Park P.G. and Ko. J.W. (2007). Stability and robust stability for systems with a time-varying delay. Automatica, $43,1855-1858$. 
Richard J.-P. (2003). Time delay systems: an overview of some recent advances and open problems. Automatica, 39, 1667-1694.

Seuret A., Michaut F., Richard J.-P., and Divoux T. (2006). Networked control using gps synchronization. Proc. of ACC06, American Control Conf., Mineapolis, USA.

Sun Y.G. and Wang L. (2006). Stability of switched systems with time-varying delays: delay-dependent common lyapunov functional approach. Proceedings of the 2006 American Control Conference.

Xie G. and Wang L. (2005). Stabilization of switched linear systems with time-delay in detection of switching signal. Mathematical analysis and application, 305, 277-290.

Yan P. and Özbay H. (2008). Stability analysis of switched time delay systems. SIAM Journal on Control and Optimization, 47(2), 936-949.

Yue D., Tian E., and Zhang Y. (2008). A piecewise analysis method to stability analysis of linear continuous/discrete systems with time-varying delay. International Journal of Robust and Nonlinear Control.

Zhai G., Hu B., Yasuda K., and Michel A.N. (2001). Stability analysis of switched systems with stable and unstable subsystems: an average dwell time approach. American Control Conference. 